\title{
Hydrophobins are required for conidial hydrophobicity and plant root colonization in the fungal biocontrol agent Clonostachys rosea
}

\author{
Mukesh K Dubey*, Dan Funck Jensen and Magnus Karlsson
}

\begin{abstract}
Background: Filamentous fungi produce small cysteine rich surface active amphiphilic hydrophobins on the outer surface of cell walls that mediate interactions between the fungus and the environment. The role of hydrophobins in surface hydrophobicity, sporulation, fruit body formation, recognition and adhesion to host surface and virulence have been reported. The aim of the present study was to characterize the biological function of hydrophobins in the fungal biocontrol agent Clonostachys rosea in order to understand their potential roles in biocontrol mechanisms.

Results: Based on the presence of hydrophobin domains, cysteine spacing patterns and hydropathy plots, we identified three class II hydrophobin genes in C. rosea. Gene expression analysis showed basal expression of Hyd1, Hyd2 and Hyd3 in all conditions tested with the exception of induced Hyd1 expression in conidiating mycelium. Interestingly, up-regulation of Hyd1, Hyd2 and Hyd3 was found during C. rosea self interaction compared to interactions with the fungal plant pathogens Botrytis cinerea or Fusarium graminearum in dual culture assays. Phenotypic analysis of C. rosea deletion and complementation strains showed that Hyd 1 and Hyd3 are jointly required for conidial hydrophobicity, although no difference in mycelia hydrophobicity was found between wild type (WT) and mutant strains. Interestingly, mutant strains showed increased growth rates, conidiation and enhanced tolerances of conidia to abiotic stresses. Antagonism tests using in vitro dual culture and detached leaf assays showed that the mutant strains were more aggressive towards B. cinerea, F. graminearum or Rhizoctonia solani, and that aggression was partly related to earlier conidial germination and enhanced tolerance of mutant strains to secreted fungal metabolites. Furthermore, in vitro Arabidopsis thaliana root colonization assays revealed reduced root colonization ability of the $\Delta H y d 3$ strain, but not for the $\Delta H y d 1$ strain. Furthermore, enhanced root colonization ability for the $\Delta H y d 1 \Delta H y d 3$ strain was found in comparison to WT.
\end{abstract}

Conclusions: These results show a role for hydrophobins in conidial hydrophobicity, control of conidial germination under stress conditions, and in root colonization in C. rosea. However, functional studies of Hyd2 remains to be performed in order to fully assess the role of hydrophobins in C. rosea.

Keywords: Antagonism, Biocontrol, Clonostachys rosea, Filamentous fungi, Gene knockout, Hydrophobins

\section{Background}

Hydrophobins are small secreted proteins, produced only by filamentous fungi, which forms amphipathic layers on the outer surface of fungal cell walls $[1,2]$. The hydrophobic side of the amphipathic layer is exposed to the outside environment, while the hydrophilic side is directed towards cell wall polysaccharides [1,2]. Hydrophobins are

\footnotetext{
* Correspondence: mukesh.dubey@slu.se

Uppsala BioCenter, Department of Forest Mycology and Plant Pathology,

Swedish University of Agricultural Sciences, Box 7026, 75007 Uppsala, Sweden
}

characterized by the presence of eight conserved cysteine (Cys) residues in a typical pattern [1-3]. Apart from this, they show very limited amino acid sequence similarity with each other. The Cys residues form four intramolecular disulphide bridges suggested to prevent selfassembly of the hydrophobins in the absence of a hydrophilic-hydrophobic interface [1,2]. Based on distinct hydropathy patterns and the type of layer they form, hydrophobins are divided in to two classes [1-3]. Recent bioinformatic analyses have identified an intermediate class of hydrophobins in Trichoderma and Aspergillus 
species [4,5]. Class I hydrophobins form amyloid-like rodlets that are highly insoluble in water, organic solvents and detergents like SDS and require strong acids for solubilisation, while amphipathic monolayers formed by class II hydrophobins lack the fibrillar rodlets and can be dissolved in aqueous organic solvents and detergents $[1,2]$. Another distinguishing characteristic of hydrophobins is the specific spacing patterns of conserved Cys residues; the consensus Cys spacing pattern $\mathrm{C}-\mathrm{X}_{5-10}-\mathrm{CC}-\mathrm{X}_{33-41}-\mathrm{C}$ $\mathrm{X}_{16-25}-\mathrm{C}-\mathrm{X}_{5}-\mathrm{CC}-\mathrm{X}_{13-17}-\mathrm{C}$ of Class I differs from the consensus Cys spacing pattern $C-\mathrm{X}_{9-10}-\mathrm{CC}-\mathrm{X}_{11}-\mathrm{C}-\mathrm{X}_{16}-\mathrm{C}-\mathrm{X}_{8-9^{-}}$ CC-X ${ }_{10}-\mathrm{C}$ of Class II [3-5].

Hydrophobins act as natural surfactants and reduce the surface tension of the medium, and perform a variety of biological functions in the life cycle of filamentous fungi. These include formation of a protective layer surrounding the hyphae and sexual structures, development of aerial hyphae, sporulation and spore dispersal, and fruit body formation [1-3]. In addition, hydrophobins mediate contact and communication between the fungus and its environments; that can include recognition and adhesion to host surfaces, and development of penetration structures during pathogenic and symbiotic interactions [3,6,7]. Hydrophobin MPG1 of the rice blast fungus Magnaporthe oryzae is necessary for leaf surface attachment and appresorium formation [8], while another hydrophobin MHP1, of the same fungus is involved in the late stage of pathogenesis [9]. In the entomopathogenic fungus Beauveria bassiana, deletion of hydrophobin genes results in decreased spore hydrophobicity and adhesion, loss of water-mediated dispersal, and lowered virulence to insects [10]. Similarly, in another entomopathogenic fungus, Metarhizium brunneum, characterization of hydrophobins showed their role in conidiation, hydrophobicity, pigmentation and virulence [11]. Contrary to these reports, functional characterization of hydrophobins in Fusarium verticilloides does not indicate a role of these proteins in growth, infection or mycelium hydrophobicity [12]. Similar results are reported for Botrytis cinerea where deletion mutants of hydrophobin genes does not display any phenotypic differences compared to the wild type (WT) strain [13].

The fungus Clonostachys rosea is a ubiquitous soil borne ascomycete known for its antagonistic abilities against a wide range of plant pathogens [14-18], and for its entomopathogenic and nematophagous behaviour [19-21]. The modes of action of $C$. rosea as a biological control agent (BCA) are not fully known, although mycoparasitism, competition for nutrients, and secondary metabolite production are suggested to play significant roles [14,18,22]. Furthermore, C. rosea can rapidly colonize outer and inner root surfaces (epidermal and cortical cells) of plants like carrot, barley, cucumber and wheat [23,24], which results in induced defence responses [25]. Hydrophobins in mycoparasitic Trichoderma spp, are suggested to be involved in hyphal development, sporulation, and plant root attachment and colonization [26-28].

The current study aims to understand the biological function of hydrophobins in C. rosea with emphasis on its role in fungal growth and development, antagonism, and interactions with plants. Our results showed induced expression of C. rosea Hyd1, Hyd2 and Hyd3 in dual cultures during self interaction in comparison to interaction with the phytopathogenic fungi $B$. cinerea and $F$. graminearum. In addition, $H y d 1$ showed significant upregulation in conidiating mycelium, although a basal expression of C. rosea Hyd1, Hyd2 and Hyd3 was observed in all conditions tested. By generating individual Hyd1 and Hyd3 deletion ( $\Delta H y d 1 ; \Delta H y d 3)$, complementation $(\Delta H y d 1+; \Delta H y d 3+)$ and Hyd1, Hyd3 double deletion $(\Delta H y d 1 \Delta H y d 3)$ strains, we probed the roles of two $C$. rosea hydrophobins in conidial hydrophobicity and plant root colonization.

\section{Results}

Identification and phylogenetic analysis of $C$. rosea hydrophobins

Blast searches against a C. rosea strain IK726 draft genome database using a total of 35 class I, class Ia (the intermediate class) and class II hydrophobin amino acid sequences from Trichoderma spp. [29], identified three genes with an E-value $\leq 1 \times 10^{-5}$. The presence of additional hydrophobin gene/s in $C$. rosea genome cannot be excluded, as the short hydrophobin genes may be problematic to predict. Identification of start and stop codons, determination of exon-intron boundaries and open reading frames (ORFs) were done manually, and were further validated by cDNA sequencing. The resulting genes were named $H y d 1, H y d 2$ and $H y d 3$. The $H y d 1$, $H y d 2$ and $H y d 3$ sequences are submitted to GenBank with accession numbers KF834267, KF834268, KF834269, respectively. The 267 bp Hyd1 ORF was interrupted by a predicted 48 bp intron, the 372 bp Hyd2 ORF was interrupted by a predicted 86 bp intron, while the 300 bp Hyd3 ORF was interrupted by a predicted 62 bp intron. The predicted 88, 123 and 99 amino acid (aa) sequences of Hyd1, Hyd2 and Hyd3, respectively, all contained a 60-65 aa core structure that contained the Cys residues. The conserved domain analysis of translated aa sequences using Simple Modular Architecture Research Tool (SMART) identified a single hydrophobin_2 domain (Pfam 06766) between aa positions 21-86, 21-85 and 30-91for Hyd1, Hyd2 and Hyd3, respectively. This structure was further confirmed by InterproScan and Conserved Domain Search (CDS) analyses. Signal P predicted 16-18 aa long secretion signal peptides in the N-termini of each $C$. rosea hydrophobin. The highest similarity of Hyd1 was with cerato-ulmin of Geosmithia spp. and Ophistoma nova-ulmi (e-value 3e-07; identity 33\%), of $\mathrm{Hyd} 2$ with T. atroviride hydrophobin and 
spore related hydrophobin of $T$. viride (e-value $3 \mathrm{e}-10$; identity $41 \%$ ), and of Hyd3 with hydrophobin from Fusarium spp. (e-value 3e-32; identity 73\%). In addition, aa similarity between Hyd1, Hyd2 and Hyd3 were below $20 \%$.

Hyd1 and Hyd2 contained eight Cys in their protein sequences, while Hyd3 contained only seven as the Cys residue closest to the $\mathrm{C}$-terminus was replaced by a glutamine (Gln) (Figure 1). This replacement was similar to the T. harzianum hydrophobin QID3 that also contained seven Cys [30], although Hyd3 did not show the extended N-terminus of QID3. The Cys spacing of Hyd1, Hyd 2 and Hyd3 conformed to the pattern of Class II (Figure 1). Furthermore, the hydropathy patterns of Hyd1, Hyd 2 and Hyd3 were all indicative of class II hydrophobins (data not shown). Taken together, these analyses suggest that $C$. rosea $\mathrm{Hyd} 1, \mathrm{Hyd} 2$ and $\mathrm{Hyd} 3$ encode putative class II hydrophobins.

A phylogenetic tree was constructed with Hyd1, Hyd2 and Hyd3 together with class II hydrophobins from Trichoderma spp. and additional representatives of known class II hydrophobins (Additional file 1: Table S1). The result from the phylogenetic analysis showed that Hyd1, $\mathrm{Hyd} 2$ and Hyd3 do not represent recent gene duplicates as they clustered in different parts of the tree (Figure 2).

\section{Expression analysis of Hyd1, Hyd2 and $\mathrm{Hyd} 3$}

Quantitative PCR (qPCR) was used to analyse the expression pattern of $C$. rosea hydrophobins. In relation to glucose, no significant expression changes in $H y d 1, H y d 2$ or Hyd3 expression were found in SMS culture representing carbon limitation $(\mathrm{C}$ lim) or nitrogen limitation $(\mathrm{N}$ lim) (Figure 3A). Gene expression analysis was performed on RNA extracted from germinated conidia (GC), mycelium $(\mathrm{M})$, conidiating mycelium $(\mathrm{CM})$, aerial hyphae $(\mathrm{AH})$, and during interaction with barley roots $(\mathrm{Cr}-\mathrm{Br})$. In relation to GC, a significant $(P \leq 0.03)$ induction in $H y d 1$ expression was found in $\mathrm{M}, \mathrm{CM}$ and $\mathrm{AH}$ (Figure $3 \mathrm{~B}$ ). In addition, $\mathrm{CM}$ showed significant $(P=0.03)$ induced expression of $H y d 1$ in comparison with $\mathrm{M}, \mathrm{AH}$ and $\mathrm{Cr}-\mathrm{Br}$ (Figure 3B). No significant changes in expression of $\mathrm{Hyd} 2$ or $\mathrm{Hyd} 3$ were found in any of the developmental conditions tested or during root interaction (Figure 3B). For hydrophobin gene expression during interactions between $C$. rosea and B. cinerea or F. graminearum, RNA was extracted from the mycelium harvested at different stages of interaction as described in methods section. Transcript levels of $C$. rosea hydrophobins were found to be significantly induced $(P \leq 0.013)$ at all stages of self interaction in comparison with interspecific interactions (Figure $3 \mathrm{C}$ ). No significant difference in expression of C. rosea hydrophobin genes were found between different stages of interaction with either of prey fungus except the significant $(P \leq 0.02)$ induced expression of Hyd1 at contact and after contact stage in comparison to before contact stage during the interaction with $B$. cinerea, but not with the $F$. graminearum (Additional file 1: Figure S1). An additional observation was that a basal expression of all C. rosea hydrophobin genes was observed in all tested conditions.

\section{Generation of Hyd1, Hyd3 and Hyd1Hyd3 deletion and complementation strains}

Single Hyd1 and Hyd3 deletion mutants were generated by replacing $H y d l$ and $H y d 3$ with the hygromycin resistance gene selection cassette (hygB) by Agrobacterium tumefaciens mediated transformation (ATMT). A double $\Delta H y d 1 \Delta H y d 3$ deletion strain was constructed by replacing Hyd3 with the nourseothricin resistance gene selection cassette (nat1) in a $\Delta H y d 1$ strain. Despite several attempts of transformation and screening of more than 200 hygromycin resistance colonies, we failed to generate a $\mathrm{Hyd} 2$ deletion mutant. Successful gene replacement in mitotically stable putative mutants was confirmed by PCR as described previously [31-33] using primers located within the hygB/nat1 cassettes together with primers located upstream and downstream of the construct (Additional file 1: Figure S2A, E, I). The expected size of PCR fragments were amplified in $\Delta H y d 1, \Delta H y d 3$ and $\Delta H y d 1 \Delta H y d 3$ strains, while no amplification was observed in wild type (WT) (Additional file 1: Figure S2B, F, J). The complete deletion of Hydland Hyd3 was further confirmed by PCR

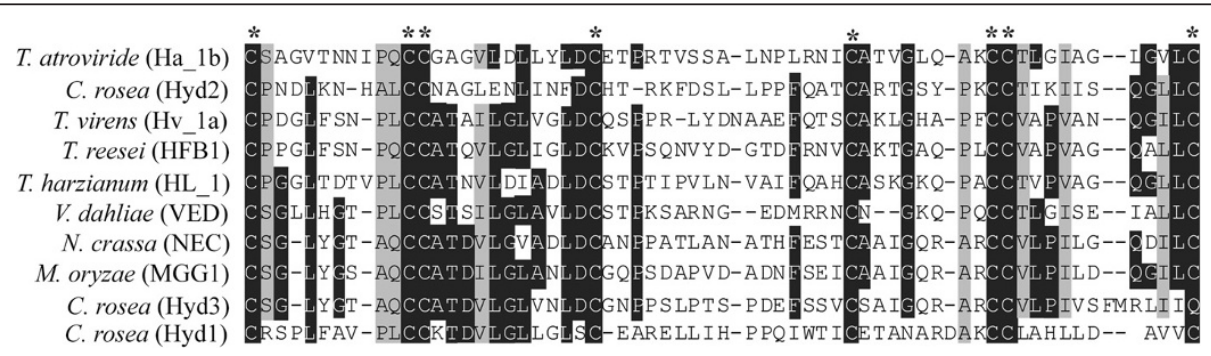

Figure 1 Sequence alignment of $C$. rosea hydrophobins. Amino acid sequence alignment of $C$. rosea hydrophobins with class II hydrophobins from Trichoderma spp. and additional representatives of known class II hydrophobins. The amino acid sequences from first Cys to eight Cys residues were used for the alignment. Conserved residues in a column are indicated in white and boxed in black; two different conserved residues in a column are highlighted by grey boxes; gaps are indicated by dashes. Conserved Cys residues are indicated by asterisks. 


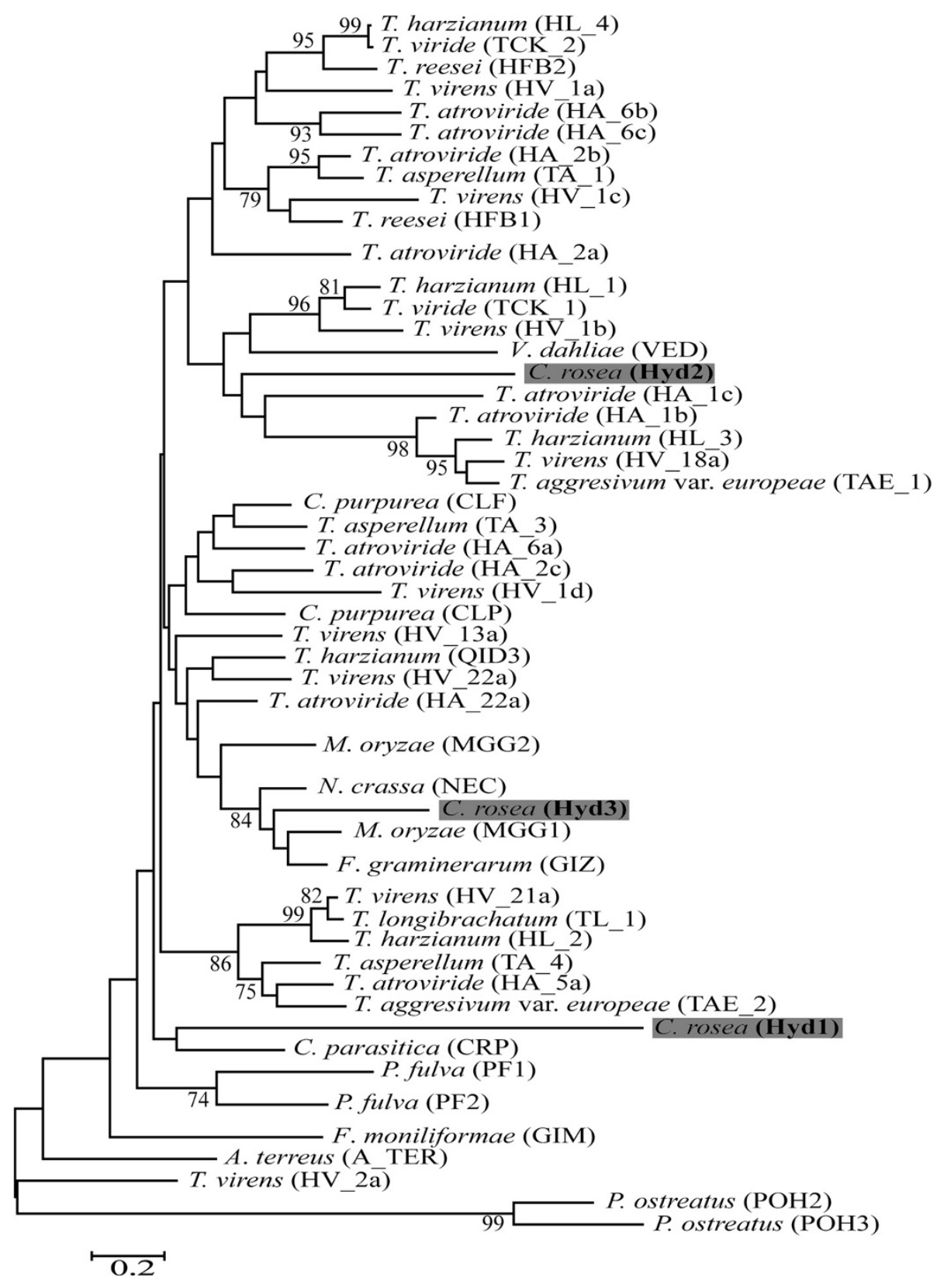

Figure 2 Phylogenetic analysis of $\boldsymbol{C}$. rosea hydrophobins. Phylogenetic analysis of class II hydrophobins using maximum likelihood methods implemented in PhyML-aBayes. Pleurotus ostreatus hydrophobins are used as out group. Branch support values (bootstrap proportions $\geq 70 \%)$ are associated with nodes. The bar marker indicate the number of amino-acid substitutions.

amplification of fragments of expected size using primer pairs located outside the construct borders, from mutant and WT strains (Additional file 1: Figure S2B, F, J). Furthermore, reverse transcriptase PCR (RT-PCR) experiments using primers specific to $H y d l$ and $H y d 3$ sequences demonstrated the complete loss of Hyd1 and Hyd3 transcript in each individual and double deletion mutants (Additional file 1: Figure S2C, G, K).

Single Hyd1 and Hyd3 deletion mutants were complemented with WT Hyd1 and $H y d 3$ genes respectively, through ATMT. Successful integration of the Hyd1-comp and Hyd3-comp vectors (including the nat1 selection cassette) in mitotically stable mutant was confirmed by PCR amplification of nat1 (data not shown). RT-PCR from randomly selected nat 1 positive $H y d 1$ and $H y d 3$ complemented $(\Delta H y d 1+; \Delta H y d 3+)$ strains using Hyd1and $H y d 3$-specific primer pairs demonstrated restored $H y d 1$ and $H y d 3$ transcription while no transcripts were detected in the parental deletion strains (Additional file 1: Figure S2D, H). 


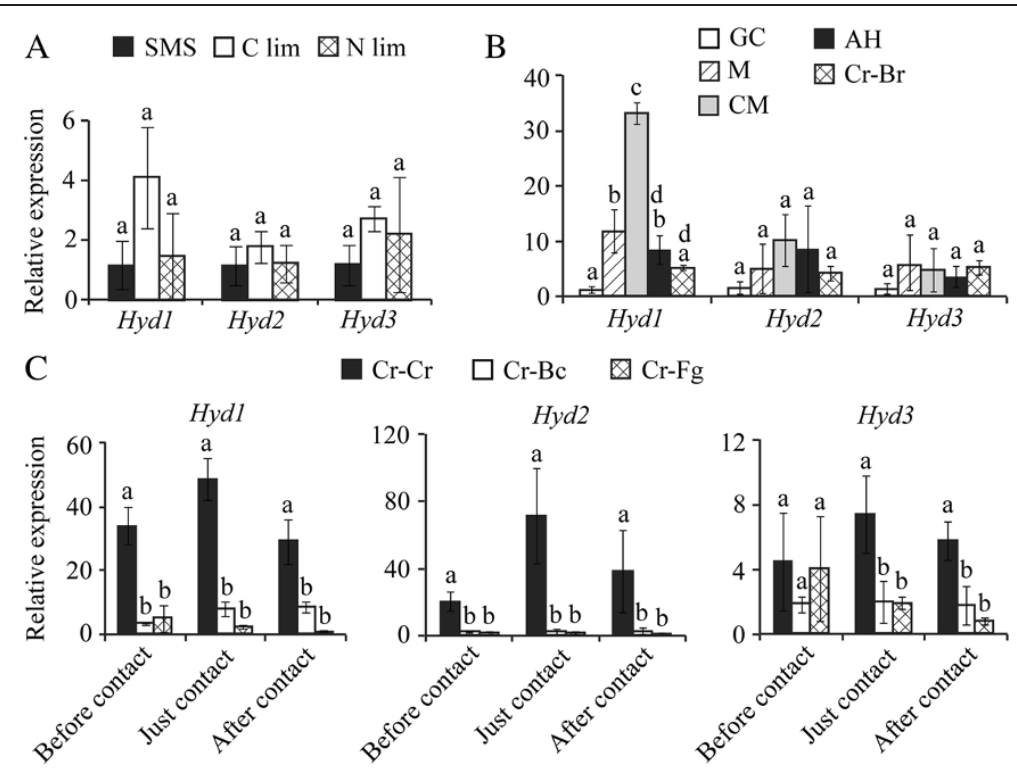

Figure 3 Expression analyses of hydrophobin genes in C. rosea. A: Total RNA was extracted from mycelia $24 \mathrm{~h}$ post incubation in submerged shake flask culture in glucose, C lim and N lim medium. B: Total RNA was extracted from mycelia of different developmental stages like germinating conidia (GC), vegetative mycelium (M), Conidiated mycelim (CM), aerial hyphae $(A H)$ and post five days interaction with barley roots $(\mathrm{Cr}-\mathrm{Br})$. C: gene expression analysis during different stages of interaction with B. cinerea $(\mathrm{Cr}-\mathrm{BC})$ or $\mathrm{F}$. graminearum $(\mathrm{Cr}-\mathrm{Fg})$. C. rosea confronted with itself was used as control ( $\mathrm{Cr}-\mathrm{Cr}$ ). Expression levels for Hyd1, Hyd2 and Hyd3 was normalized by tubulin expression, using the formula described by Pfaffl [52]. Error bars represent standard deviation based on 3 biological replicates. Different letters indicate statistically significant differences $(P \leq 0.05)$ within experiments based on the Tukey-Kramer test.

Effects of Hyd1 and Hyd3 deletion on colony morphology, growth rate, conidiation, hydrophobicity, and secreted protein concentration

No difference in colony morphology was found between WT and deletion mutants (data not shown). All deletion strains showed significantly $(P<0.001)$ increased growth rate and conidiation on potato dextrose agar (PDA) medium in comparison to WT, although no differences were detected between single deletion strains or between single and double deletion strains (Figure 4A, B). Complementation strains $\Delta H y d 1+\Delta H y d 3+$ showed partial restoration of normal conidiation levels (Figure 4B).

Hydrophobicity of WT and mutant strains were tested by carefully placing $10 \mu \mathrm{l}$ water or SDS $(0.2 \%$ or $0.5 \%)$ droplets onto the surface of non-conidiating mycelia (3 days post inoculation on PDA). All droplets remained on the surface of mycelium and no visible difference in shape or contact angle of droplets was found in between WT and mutant strains even up to overnight incubation in closed Petri-dishes at room temperature. Similar results were obtained when conidiated mycelia (10 days post inoculation) were used. Conidial surface hydrophobicity was further analysed by using an assay for microbial adhesion to hydrocarbons (MATH) [34]. The MATH assay showed no difference in hydrophobicity index between WT and single deletion mutants; however conidia of the double deletion mutant showed significant $(P<0.001)$ reduction in hydrophobicity index (Figure 4C). In addition, unlike the WT, $\Delta H y d 1$ and $\Delta H y d 3$, conidia from the $\triangle H y d 1 \Delta H y d 3$ strain formed cell aggregates when harvested in water (Additional file 1: Figure S3).

To analyse total protein secretion, protein concentrations were determined in culture filtrates of WT and mutant strains grown in liquid potato dextrose broth (PDB) medium. Results showed a significant $(P \leq 0.004)$ $9 \%$ reduction in protein concentration in $\Delta H y d 1 \Delta H y d 3$ culture filtrates compared to WT or single deletion strains, while no differences were observed in between WT and $\Delta H y d 1$ or $\Delta H y d 3$ strains (Figure 4D).

\section{Effect of Hyd1 and Hyd3 deletion on abiotic stress tolerance} Susceptibility of WT and mutant strains to various abiotic stress conditions were tested on PDA plates containing $\mathrm{NaCl}$, sorbitol, SDS, or caffeine. No significant differences in growth rate were recorded between mutant and WT strains on any of the tested stress media, except for significantly $(P=0.028)$ increased growth rate of the double deletion mutant $\Delta H y d 1 \Delta H y d 3$ on PDA containing $\mathrm{NaCl}$ (Additional file 1: Figure S4). Significant $(P<0.001)$ increases in conidial germination rates (>90\%) were recorded in mutant strains in comparison with WT (55\% to $60 \%$ ) on all tested abiotic stress media, although no differences were found between WT and mutant strains on control PDA medium (Figure 5A). In another set of experiments we assayed the conidial susceptibility to cold. After 3, 6 or 9 days of incubation at 


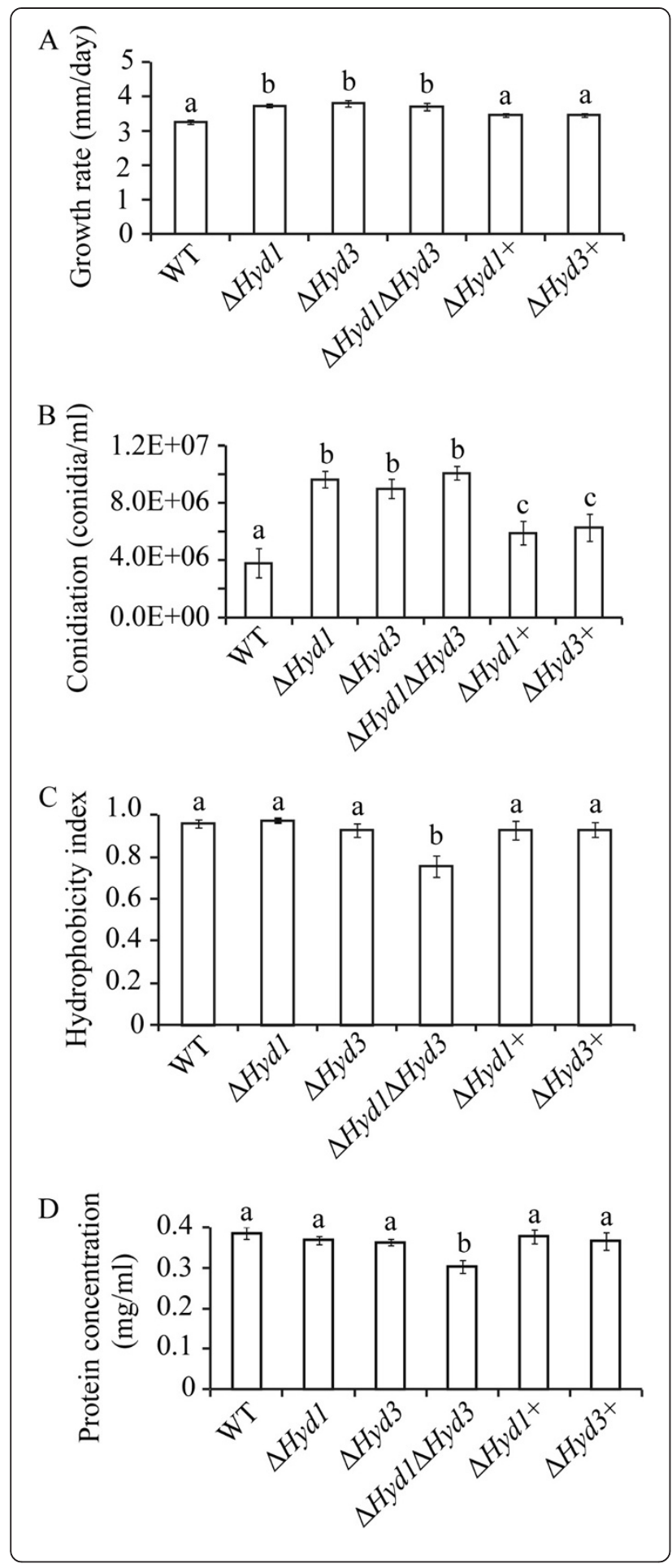

Figure 4 Phenotypic characterizations of $C$. rosea hydrophobin mutants. A: Growth rate of WT, mutants and complemented strain on PDA medium. Strains were inoculated on solid agar medium, incubated at $25^{\circ} \mathrm{C}$ and the growth diameter was recorded 5 days post inoculation. B: Conidiation of WT, mutants and complemented strain on PDA medium 10 days post inoculation. Conidia were harvested in equal volume of water and number was determined using a Bright-Line haemocytometer as per instruction of manufacturer. C: Cell surface hydrophobicity of WT, deletions and complemented strains conidia as determined by microbial adhesion to hydrocarbon (MATH) assay. D: Total extracellular protein concentration of WT deletions and complemented strains. Culture filtrates of 10 days grown fungal strains were used for protein precipitation. Error bars represent standard deviation based on 3 biological replicates. Different letters indicate statistically significant differences $(P \leq 0.05)$ based on the Tukey-Kramer test. Experiments were repeated two times with same results.

$4^{\circ} \mathrm{C}$, only $63 \%, 33 \%$ or $30 \%$ respectively, of WT conidia germinated after placing them on PDA medium for $16 \mathrm{~h}$ at $25^{\circ} \mathrm{C}$. In contrast, similarly treated conidia of mutants strain showed significantly $(P<0.001)$ higher germination rates $(82 \%, 64 \%$ and $56 \%$ ) (Figure $5 \mathrm{~B})$. However, no differences in conidial germination between either of single or double deletion mutants were found in any of the stress condition tested (Figure 5).

\section{Deletion of Hyd1 and Hyd3 did not affect Hyd2 expression} In order to examine whether or not deletion of $H y d 1$ and $H y d 3$, individually or simultaneously, affects the expression pattern of $H y d 2$, RNA was extracted from conidiating mycelium of WT and mutant strains grown on PDA plates. Gene expression analysis revealed no significant difference in $H y d 2$ expression between WT and either single or double deletion strains (Additional file 1: Figure S5).

In vitro assay to test the antagonistic ability of $C$. rosea strains The $\Delta H y d 1, \Delta H y d 3$, and $\Delta H y d 1 \Delta H y d 3$ strains overgrew $B$. cinerea, F. graminearum and Rhizoctonia solani faster than the WT in plate confrontation assays (Figure 6A). The complemented strains $\Delta H y d 1+\Delta H y d 3+$ showed partial restoration of WT behaviour. Furthermore, in order to understand the tolerance of $C$. rosea strains to the secreted metabolites from the fungal prey, a secretion assay was performed. Growth rates of deletion strains were significantly $(P<0.001)$ higher than the WT when grown on agar plates where B. cinerea, F. graminearum or $R$. solani were pregrown (Figure 6B). In addition, the double deletion strain $\Delta H y d 1 \Delta H y d 3$ showed significantly $(P \leq 0.05)$ higher growth rate compared to the either single deletion mutant (Figure 6B). Similarly to the plate confrontation assay, $\Delta H y d 1+$ and $\Delta H y d 3+$ strains showed partial restoration of WT growth rates.

In another set of experiments, mycelial biomass of $B$. cinerea, F. graminearum and $R$. solani was measured in sterile-filtered culture filtrates of $C$. rosea WT and deletion strains. A significantly $(P<0.001)$ higher biomass 

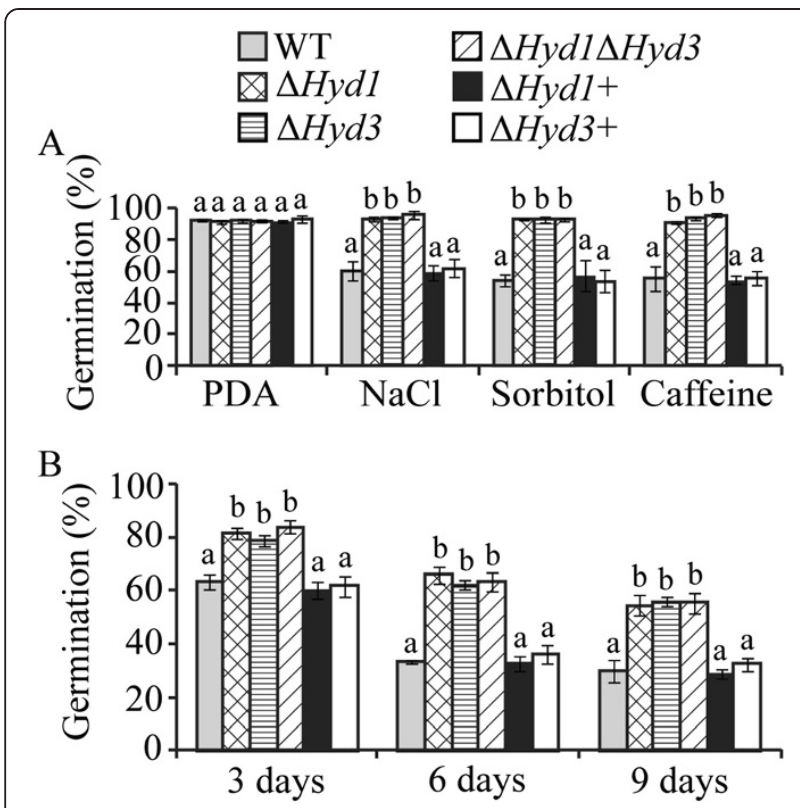

Figure 5 Abiotic stress tolerances of $C$. rosea WT and mutant strains. A: Frequency of conidia germination on medium containing $\mathrm{NaCl}$, sorbitol, SDS, or caffeine as abiotic stress agents. Conidia spread on PDA plate were served as control. B: Frequency of conidia germination after cold shock at $4^{\circ} \mathrm{C}$ for 3 days, 6 days or 9 days. C. rosea WT, mutants and complementation strains conidia were spread on agar plates and frequency of conidial germination was determined by counting two hundred to three hundred conidial germ-tubes or conidia under microscope for each treatment. Each experiment was repeated two times. Error bars represent standard deviation based on 3 biological replicates. Different letters indicate statistically significant differences $(P \leq 0.05)$ based on the Tukey-Kramer test.

production of B. cinerea, F. graminearum and $R$. solani was recorded when grown in culture filtrates of hydrophobin deletion strains compared with WT culture filtrate (Figure 6C). No differences in fungal biomass production were found between culture filtrates of either single or double mutant strains (Figure 6C).

\section{Assessment of antagonistic activity of $C$. rosea strains using a detached leaves assay}

A significant $(P<0.001)$ reduction in necrotic lesion area was measured on leaves preinoculated with $C$. rosea WT compared to control leaves where only $B$. cinerea was inoculated (Figure 7). In addition, in leaves preinoculated with $\Delta H y d 1, \Delta H y d 3$, or $\Delta H y d 1 \Delta H y d 3$ strains, necrotic lesion areas were significantly $(P<0.001)$ less severe than those observed in WT preinoculated leaves. No difference in necrotic lesion areas were found between leaves preinoculated with either single or double deletion strains (Figure 7).

Assessment of $C$. rosea strains for root colonization ability Arabidopsis thaliana roots, grown on MS plates, were inoculated with $C$. rosea conidia and allowed to interact for 5 days. Water inoculated roots were used as control. After surface sterilization, colonization levels were determined by counting colony forming units (cfus). No significant differences in root colonization ability were recorded between WT and the $\Delta H y d 1$ strain. In contrast, root colonization by the $\Delta H y d 3$ strain was significantly $(P<0.001)$ reduced (Figure 8$)$. Interestingly, the double deletion $\Delta H y d 1 \Delta H y d 3$ strain showed increased $(P<0.001)$ colonization ability compared to WT or single deletion strains (Figure 8).

\section{Discussion}

Filamentous fungi generally contain multiple hydrophobin genes, which play important roles in fungal growth, development and environmental communication $[1,2,6,7]$. We identified only 3 class II hydrophobin genes in the genome of the mycoparasite $C$. rosea. This is in strong contrast with the closely related mycoparasites $T$. atroviride and $T$. virens that contain high numbers (10 and 9 respectively) and diversity of class II hydrophobins [29]. This indicate important ecological differences between C. rosea and Trichoderma spp., and emphasize that different mycoparasites may rely on different mechanisms of interaction. The expansion of the hydrophobin gene family in Trichoderma spp. is hypothesized to help the fungus to attach to the hyphae of a broad range of asco- and basidiomycetes [29].

The high expression of $H y d 1$ in conidiating mycelia in comparison with germinating conidia indicates that Hyd1 may have a role during conidiophore development. This is consistent with the expression pattern of hyd1 in M. anisoplia where expression is low in germinating conidia and high in mycelium with conidiophores [35]. The expression, but lack of regulation, of Hyd1, Hyd2 and Hyd3 on different nutrient regimes, and between developmental stages of $H y d 2$ and $H y d 3$, indicate a constitutive role of the corresponding proteins in C. rosea. Constitutive roles of hydrophobins in fungal growth and development are reported in many species $[6,7,36]$. However, certain hydrophobins from Trichoderma spp. and M. brunneum are regulated by nutritional conditions and between different life cycle stages $[5,11,28,37]$.

Expression levels of $H y d 1, H y d 2$ and $H y d 3$ are repressed in $C$. rosea during interactions with $B$. cinerea and $F$. graminearum, which is consistent with the expression pattern of $T$. atroviride hydrophobin genes $h f b-1 b$, hfb$2 c$ and $h f b-6 a$ [37]. This may suggest that Hyd1, Hyd2 and Hyd3 are not involved in protecting hyphae from recognition by other organisms [6,7]. Alternatively, the data can also be interpreted as an induction during $C$. rosea self interaction that may suggest a role for Hyd1, Hyd2 and $\mathrm{Hyd} 3$ in intraspecific signalling or hyphal fusion. Hydrophobins that are known to be involved in interactions 


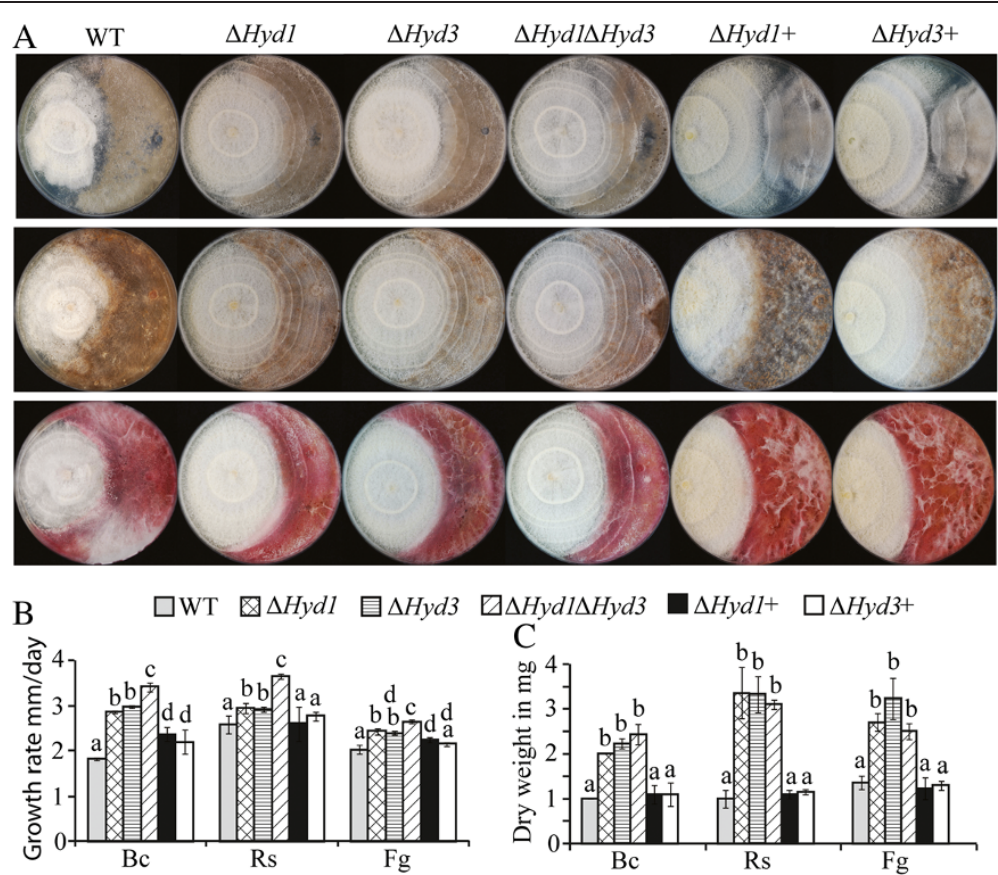

Figure 6 Antagonism analyses of C. rosea strains. A: Plate confrontation assay against B. cinerea (Uppar lane), R. solani (middle lane) and F. graminearum (lower lane). Agar plugs of C. rosea (left side in the plate) strains and B. cinerea, R. solani or F. graminearum (right side in the plate) were inoculated on opposite sides in $9 \mathrm{~cm}$ diameter agar plates and incubated at $25^{\circ} \mathrm{C}$. The experiment was performed in 3 replicates and photographs of representative plates were taken 20 days post inoculation. B: Tolerance of $C$. rosea strains to the secreted metabolites of $B$. cinerea (Bc), R. solani (Rs) and F. graminearum (Fg). Agar plugs were inoculated on PDA plates covered with cellophane and incubated at $25^{\circ} \mathrm{C}$ in darkness. After reaching to the end of plate the colony was removed together with the cellophane disc. Plates were re-inoculated with a C. rosea WT, $\Delta H y d 1$, $\Delta H y d 3, \Delta H y d 1 \Delta H y d 3$, and $\Delta H y d 1+, \Delta H y d 3+$ agar plug, incubated at $25^{\circ} \mathrm{C}$ and linear growth was recorded daily. C: Secretion assay of $C$. rosea strain. Fungal strains were grown in potato dextrose broth for 10 days at $25^{\circ} \mathrm{C}$. Culture filtrates was collected after removing mycelia mass and were inoculated with B. cinerea (Bc), R. solani (Rs) or F. graminearum (Fg) agar plug. Biomass production in culture filtrates was analysed by determining mycelial dry weight post 3 days of inoculation. Error bars represent standard deviation based on 3 biological replicates. Different letters indicate statistically significant differences $(P \leq 0.05)$ within experiments based on the Tukey-Kramer test.

with plant leaves and roots are usually highly expressed during these conditions $[8,9,28]$. Therefore, the low expression of the 3 C. rosea hydrophobin genes during barley root colonization indicates that the corresponding proteins may not be necessary for root adhesion and colonization.

Deletion of hydrophobin genes from different fungal species often results in variable and sometimes contradicting phenotypes. This is a reflection of the birth-anddeath type of evolution of the hydrophobin gene family [29], which results in functionally diverse proteins with many species specific members. This is evident for Hyd1 and Hyd3 in C. rosea as gene deletions results in increased growth rate and sporulation, which is in contrast to the reduced sporulation in T. reesei, $M$. oryzae and $M$. brunneum due to deletion of the hydrophobin genes HFB2 [26], MPG1 and MHP1 [8,9] and hyd1, hyd2 and hyd3 [11], respectively. The situation is even more complicated as deletion of HCf-1 and HCf-2 in Cladosporium fulvum [34], cpph1 in Claviceps purpurea [38] and hfb1 in T. reesei [26] results in no differences in sporulation in comparison with the WT strain.
Deletion of $H y d 1$ or $H y d 3$ does not influence mycelial hydrophobicity in C. rosea, which is consistent with previous reports in C. purpurea, M. brunneum, F. verticilloides and B. cinerea [11-13,38]. However, it seems that Hyd 1 and Hyd3 are jointly required for conidial hydrophobicity and dispersal, as the conidia from the double deletion mutant $\Delta H y d 1 \Delta H y d 3$ clump together in solution and have lower hydrophobicity index than the WT. Similar phenotypes are repeatedly reported from many different species $[8,9,11,12,34,39]$. Furthermore, deletion of $H y d 1$ and $H y d 3$ does not influence the expression levels of $H y d 2$, which suggests that $H y d 2$ is subject to different regulatory signals than $H y d 1$ and $H y d 3$. Failure to delete $H y d 2$ despite several trials may suggest an essential function of the corresponding protein.

Hyd1 and Hyd3 do not appear to be involved in protection of the $C$. rosea mycelium during abiotic stress conditions. In contrast, higher conidial germination rates during abiotic stress conditions in Hyd1 and Hyd3 mutants suggests that these hydrophobins inhibit conidial germination in environments not suitable for mycelial 


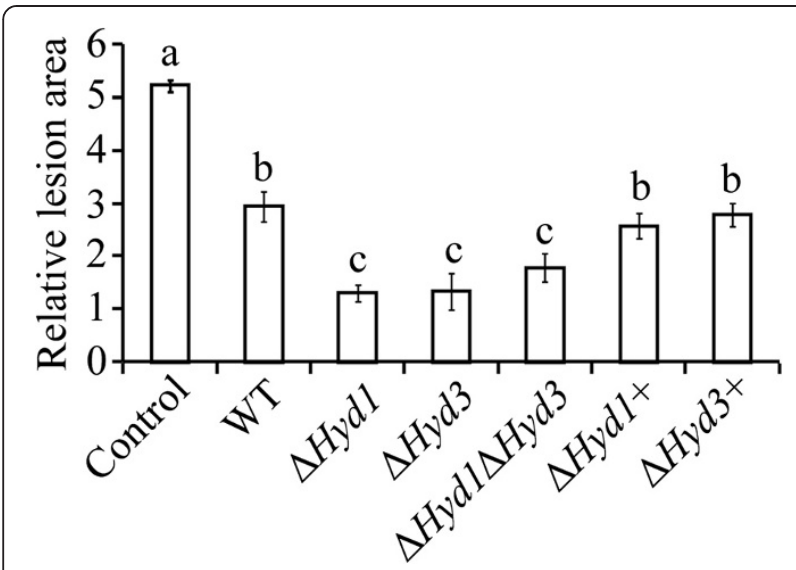

Figure 7 Measurement of $B$. cinerea necrotic lesions on detached leaves of $A$. thaliana plants. The leaves were inoculated with $C$. rosea strains 30 minute before application of $B$. cinerea and allowed to interact for $56 \mathrm{~h}$. Only pathogen inoculated leaves were used as control. Necrotic lesion area was measured under the microscope using DeltaPix camera and software. Error bars represent standard deviation based on 3 biological replicates. Different letters indicate statistically significant differences $(P \leq 0.05)$ within experiments based on the Tukey-Kramer test.

growth. Similar results are shown previously in $M$. oryzae and the entomopathogenic fungus $B$. bassiana against thermal stress $[9,10]$. Hence, under unfavourable conditions hydrophobins may act as a sensor for the conidial germination signalling pathway and consequently protect the conidia by limiting its germination until favourable conditions are prevail [10].

The increased growth rate of $H y d 1$ and $H y d 3$ deletion strains under normal conditions such as PDA, may explain the faster overgrowth of the fungal prey in plate

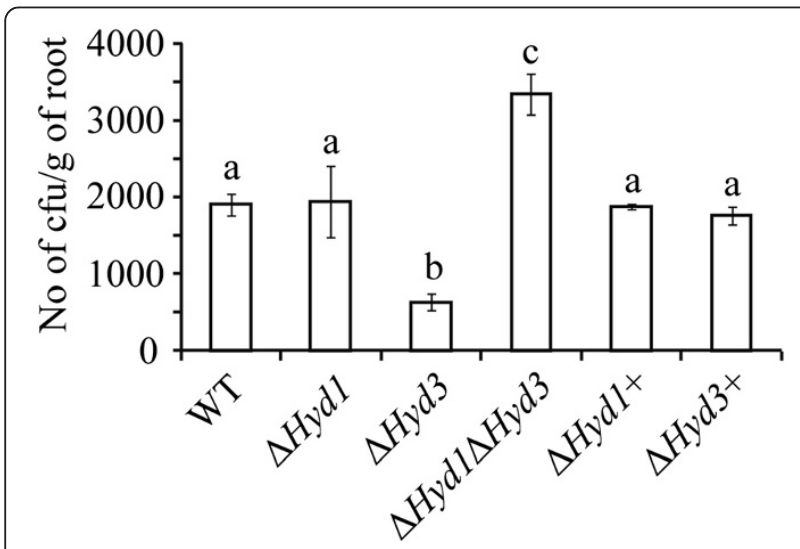

Figure 8 A. thaliana root colonization by C. rosea strains. A. thaliana roots were detached 5 days post inoculation and washed. After sterilization in $2 \% \mathrm{NaOCl}$ for 1 min, the roots were homogenized in water and serial dilutions were plated on PDA plates under sterile condition at $25^{\circ} \mathrm{C}$. Different letters indicate statistically significant differences $(P \leq 0.05)$ based on the Tukey-Kramer test. confrontations and the higher growth rate on plates previously colonized by $B$. cinerea, $F$. graminearum or $R$. solani. Similar results are reported previously in $T$. asperellum, where deletion of TasHyd1 does not reduce in vitro mycoparasitic ability [28]. Hydrophobins are highly expressed proteins that may account for up to $10 \%$ of the total amount of secreted proteins $[40,41]$. In C. rosea, deletion of both $H y d 1$ and $H y d 3$ results in a reduction of the total amount of secreted proteins. Despite this, no differences in pathogen biomass production in sterile filtered culture filtrates from single and double deletion strains are recorded. This may suggest that Hyd1 and Hyd3 do not exert a direct toxic effect on the fungal prey.

The higher conidial germination rates (under certain conditions) and higher growth rates of Hyd1 and Hyd3 deletion strains may explain the reduced necrotic lesion area, caused by $B$. cinerea, on $A$. thaliana leaves preinoculated with the mutant strains in comparison with WT preinoculated leaves. As a consequence, the C. rosea deletion strains may parasitize $B$. cinerea to a greater extent or simply outcompete it for space or nutrients. Hydrophobins in T. asperellum are reported to influence root surface attachment and intercellular root colonization [28]. Similarly, our results show that Hyd3 is needed for barley root colonization. Unexpectedly, deletion of Hyd1 in a $\Delta H y d 3$ background increases the root colonization ability. The exact mechanism responsible for this cannot be discerned based on the current data, but we may speculate that it can be related to the lower conidial hydrophobicity or the lower protein secretion of the double deletion strain compared with the $H y d 1$ and $H y d 3$ single gene deletion strains. In the entomopathogenic fungus $B$. bassiana, reduced virulence is recorded for a $\Delta h y d 1$ strain, while no effect is observed for a $\Delta h y d 2$ strain. However, the effect of the $\Delta h y d 1 \Delta h y d 2$ double deletion mutant on virulence is cumulative and lower than for the single $\Delta h y d 1$ strain [10].

\section{Conclusions}

We identified three class II hydrophobin genes and characterized their function in the fungal biocontrol agent C. rosea. Our results showed a basal expression of all three hydrophobin genes during growth and development and under nutritional stress conditions, although Hyd1 was induced during conidiation. In addition, all three genes were upregulated during self-interaction compared to the interaction with fungal prey. Deletion of C. rosea Hyd1 and Hyd3 demonstrate the involvement of the corresponding proteins in controlling conidial germination under unfavourable conditions, and the additive contribution of Hyd1 and Hyd3 to conidial hydrophobicity. Hyd3 was further shown to influence the root colonization ability of C. rosea. 


\section{Methods}

\section{Fungal strains and culture conditions}

$C$. rosea strain (WT) and mutants derived from it, $B$. cinerea strain B05.10, R. solani strain SA1 and F. graminearum strain 1104-14 were maintained on PDA (Oxoid, Cambridge, UK) medium at $25^{\circ} \mathrm{C}$. SMS medium [31] supplemented with $1 \%$ glucose was used for gene expression unless otherwise specified. Starvation for carbon (C lim), nitrogen $(\mathrm{N} \lim )$ and carbon + nitrogen $(\mathrm{C}+\mathrm{N} \lim )$ was induced as described before [31]. C. rosea mycelia for submerged liquid cultures were cultivated and harvested as described previously [31].

\section{Gene identification and sequence analysis}

The $C$. rosea strain draft genome (Karlsson et al., unpublished) was screened for the presence of hydrophobins by BLASTP analysis using amino acid sequences of $T$. aggresivum var. europeae, T. asperellum, T. atroviride, $T$. harzianum, $T$. longibrachatum, $T$. stromaticum, $T$. virens and $T$. viride hydrophobins. The protein accession numbers of hydrophobins from Trichoderma spp. (Additional file 1: Table S1) were retrieved from Kubicek et al. [29], and their amino acid sequences were retrieved from GenBank at NCBI. Presence of conserved domains were analysed with SMART [42], InterProScan [43] and CDS [44]. Presence of Cys residues in specific spacing pattern was analysed manually. Amino acid sequence alignment was performed using clustalW2 [45] with default settings for multiple sequence alignment. Signal P 4.1 [46] was used to search for signal peptide cleavage sites. Hydropathy pattern was determined with Protscale on the ExPASy proteomics server [47], using the Kyte-Doolittle algorithms and 9 aa sliding window. We generated the hydropathy pattern of Hyd1, Hyd2 and Hyd3 and compared to the hydropathy patterns of known class I (SC3 [AAA96324] from Schizophyllum commune; EAS [AAB24462] from Neurospora crassa; RodA [AFUA_5G09580] from Aspergillus fumigatus) and known class II (HFB1 [CAA92208.1] and HFBII [P79073] from T. reesei; CRP from Cryphonectria parasitica [AAA19638]) hydrophobins. The presence of conserved hydrophobin domains, Cys residues in a specific pattern, presence of signal peptide, and hydropathy plot were used as criteria for identification of hydrophobins in C. rosea.

\section{Phylogenetic analysis}

Phylogenetic analysis was performed using maximum likelihood methods implemented in PhyML-aBayes [48]. The LG amino-acid substitution model [49] was used, the proportion of invariable sites was set to 0 , and four categories of substitution rates were used. The starting tree to be refined by the maximum likelihood algorithm was a distance-based BIONJ tree estimated by the program. Statistical support for phylogenetic grouping was assessed by bootstrap analysis using 1000 replicates.
GenBank accession numbers for hydrophobin proteins used in this study for phylogenetic analysis are given in Additional file 1: Table S1.

\section{Gene expression analysis}

For gene expression analysis in different nutritional conditions (described above), mycelia were cultivated in liquid cultures following the procedure described before [31] and harvested $48 \mathrm{~h}$ post inoculation. SMS agar plates were used for gene expression analysis during different developmental stages and during interaction, except for conidial germination where liquid SMS medium was used. Developmental stages included M (mycelia harvested three days post inoculation), CM (mycelia harvested 10 days post inoculation), $\mathrm{AH}$, and GC (24 h post inoculation of conidia in liquid SMS). For interactions, C. rosea was confronted with $B$. cinerea $(\mathrm{Cr}-\mathrm{Bc})$ or $F$. graminearum $(\mathrm{Cr}-\mathrm{Fg})$ on agar plates and the growing front $(7-10 \mathrm{~mm})$ of $C$. rosea was harvested before contact (5-7 mm apart), at contact, and post $24 \mathrm{~h}$ contact. C. rosea confronted with itself $(\mathrm{Cr}-\mathrm{Cr})$ was used as control treatment. For interaction with barley roots, surface sterile seeds were germinated on sterile filter paper placed on water agar (5 seeds per replicate). C. rosea conidia $(1 \mathrm{e}+07)$ were inoculated five days post germination and were allowed to interact for five days before harvesting of roots along with fungal mycelium. Harvested samples were immediately frozen in liquid nitrogen and stored at $-80^{\circ} \mathrm{C}$.

RNA extraction from all samples was done using the Qiagen RNeasy kit following the manufacturer's protocol (Qiagen, Hilden, Germany). RNA was treated with RNasefree DNaseI (Fermentas, St. Leon-Rot, Germany) and concentrations were determined spectrophotometrically using NanoDrop (Thermo Scientific, Wilmington, DE). One or two microgram of total RNA was reverse transcribed in a total volume of $20 \mu \mathrm{l}$ using the Maxima first stand cDNA synthesis kit (Fermentas, St. Leon-Rot, Germany). Transcript levels were quantified by qPCR using the SYBR Green PCR Master Mix (Fermentas, St. Leon-Rot, Germany) in an iQ5 qPCR System (Bio-Rad, Hercules, CA) as described previously [50]. Melt curve analysis was performed after the qPCR reactions, to confirm that the signal was the result from a single product amplification. Relative expression levels for target genes in relation to tubulin expression [51] were calculated from the $\mathrm{Ct}$ values and the primer amplification efficiencies by using the formula described by Pfaffl [52]. Gene expression analysis was carried out in 3 biological replicates, each based on 2 technical replicates. Primer sequences used for gene expression analysis are given in Additional file 1: Table S2.

\section{Construction of disruption and complementation vectors} Genomic DNA was isolated using a hexadecyltrimethylammonium bromide (CTAB)-based method [53]. Phusion 
DNA polymerase (Finnzymes, Vantaa, Finland) was used for PCR amplification of a $1 \mathrm{~kb} 5^{\prime}$-flank and 3 '-flank region of the Hyd1, Hyd2 and Hyd3 genes from genomic DNA of $C$. rosea using primer pairs Hyd1 ko-1 F/1R and Hyd1 ko-2 F/2R; Hyd2 ko-1 F/1R and Hyd2 ko-2 F/2R; and Hyd3 ko-1 F/1R and Hyd3 ko-2 F/2R, respectively (Additional file 1: Table S2). The hygromycin resistance gene (hygB) cassette was amplified from the pCT74 vector [54] using the P3/P4 primer pair (Additional file 1: Table S2). The nourseothricin resistance gene (nat1) cassette was amplified from the pD-NAT1 vector [55] using the NatF/ NatR primer pair (Additional file 1: Table S2). Gateway entry clones of the purified 5 '-flank, 3 '-flank, hygB and nat cassettes PCR fragments were generated as described by the manufacturer (Invitrogen, Carlsbad, CA). The gateway $\mathrm{LR}$ recombination reactions were performed using entry plasmid of respective fragments and destination vector pPm43GW [56] to generate the disruption vectors following the conditions described by the manufacturer (Invitrogen, Carlsbad, CA).

Hyd1 and Hyd3 complementation cassettes were constructed by PCR amplification of the full-length sequence of Hyd1 and Hyd3 including $1 \mathrm{~kb}$ upstream and downstream regions from genomic DNA of $C$. rosea WT using Hyd 1 comp-F/R and Hyd3 comp-F/R primers, respectively (Additional file 1: Table S2). The amplified DNA fragments were purified and integrated into destination vector pPm43GW as described above using Gateway cloning technology to generate complementation vectors.

\section{Agrobacterium tumefaciens mediated transformation}

The disruption and complementation vectors were transformed into A. tumefaciens strain AGL-1 as described before [31-33]. A. tumefaciens mediated transformation (ATMT) was performed based on a previous protocol [57]. Transformed strains were selected on plates containing hygromycin or nourseothricin or both in the case of double deletion and complementation experiment. Putative transformants were repeatedly sub-cultured on PDA plates without the selectable agent five times, followed by re-exposure to hygromycin or nourseothricin respectively, in order to test for mitotic stability. Mitotically stable colonies were purified by two rounds of single spore isolation.

\section{Validation of transformants}

A PCR screening approach of putative transformants was performed to validate the homologous integration of the disruption cassette [31-33]. The primers used were specific to the $h y g B$ gene (P3/P4), sequences flanking the deletion construct (Hyd1-ups/ds for $\Delta H y d 1$; and Hyd3-ups/ds for $\Delta H y d 3$ ) and in combination (Hyd1-ups/ HygR_qPCR, Hyd1-ds/HygF_qPCR for $\Delta H y d 1$; and Hyd3ups/HygR_qPCR, Hyd3-ds/HygF_qPCR for $\Delta H y d 3)$. Reverse transcriptase (RT-) PCR analysis was conducted on
WT, deletion and complemented strains using RevertAid premium reverse transcriptase (Fermentas, St. Leon-Rot, Germany) and primer pairs specific for $h y g B$ (HygF_qPCR/ HygR_qPCR), nat1 (NatF_qPCR/NatR_qPCR), Hyd1 (Hyd1F/R) and Hyd3 (Hyd3-F/R) (Additional file 1: Table S2).

\section{Phenotypic analysis}

A $3 \mathrm{~mm}$ agar plug from the growing mycelial front was transferred to solid PDA, or PDA plates containing $\mathrm{NaCl}$ $(0.5 \mathrm{M})$, sorbitol $(1.5 \mathrm{M})$, SDS $(0.05 \%)$ or caffeine $(0.2 \%)$ in the case of abiotic stress analysis. Colony diameter was measured after 5 day of growth at $25^{\circ} \mathrm{C}$. Conidiation rate was determined by harvesting spores from 10 day old PDA plate cultures using a Bright-Line haemocytometer (Sigma-Aldrich, St. Louis, MO) as per instruction. For conidial susceptibility assay, conidia were harvested in sterile water from the surface of two week old PDA plates, spread on PDA plates containing $\mathrm{NaCl}(0.5 \mathrm{M})$, sorbitol $(1.5 \mathrm{M})$ or caffeine $(0.2 \%)$. Conidia spread on only PDA plates served as control. For cold stress experiments, conidia at a concentration of $1 \mathrm{e}+06 \mathrm{ml}^{-1}$ in sterile water was incubated at $4^{\circ} \mathrm{C}$ for 3 days, 6 days or 9 days and then spread on PDA plates. Frequency of conidial germination was determined post $16 \mathrm{~h}$ of spreading by counting the number of germinating and non-germinating conidia using microscope. Two hundred to three hundred conidia were counted for each treatment. Each experiment had 3 biological replicates and was repeated 2 times.

Mycelial hydrophobicity of $C$. rosea strains were assayed on PDA plates post 3 days or 10 days of inoculation using water or SDS following the procedure described before [34]. The hydrophobicity of conidia was assayed using MATH [34], and hydrophobic index was calculated following the formula described before [10]. For extracellular protein concentration determination, fungal strains were grown for 10 days in liquid $\mathrm{PDB}$ medium at $25^{\circ} \mathrm{C}$, mycelial debris were removed by filtering through four layers of Miracloth, followed by protein precipitation using an acetone precipitation protocol as described elsewhere. The protein pellets were dissolved in water and total extracellular protein concentration was determined using the quick start Bradford protein assay kit following the manufacturer's instruction (Bio-Rad, Hercules, CA).

\section{Antagonism test}

Antagonistic behaviour against phytopathogenic fungi $B$. cinerea, F. graminearum and $R$. solani was tested using an in vitro plate confrontation assay on PDA medium. An agar plug of $C$. rosea was inoculated $2 \mathrm{~cm}$ from the edge in a $9 \mathrm{~cm}$ PDA plate. After 7 days of incubation at $25^{\circ} \mathrm{C}$, a plug of $B$. cinerea, $F$. graminearum or $R$. solani was placed at equal distance to the opposite edge of plate. To test the tolerance of $C$. rosea WT, deletion or complemented strains against secreted factors of $B$. 
cinerea, F. graminearum and $R$. solani, agar plugs of phytopathogenic fungi were inoculated on PDA plates covered with cellophane and incubated at $25^{\circ} \mathrm{C}$ in darkness. The plates covered with cellophane, without inoculation, were used as control. The cellophane was removed when fungal mycelia covered the plates, followed by inoculation with $C$. rosea WT, deletion or complementation strains. Linear growth was recorded daily in 3 replicates. For secretion assay, C. rosea strains were grown for 10 days in liquid PDB medium on rotary shaker at $25^{\circ} \mathrm{C}$. Culture filtrate was collected after removing mycelia by filtering through four layers of Miracloth. The filtrate was further purified by passing through a $0.45 \mu \mathrm{M}$ pore size nylon membrane. Agar plugs of B. cinerea, $F$. graminearum or $R$. solani was inoculated in conical flasks $(50 \mathrm{ml})$ with $20 \mathrm{ml}$ culture filtrate and incubated at $25^{\circ} \mathrm{C}$ under constant shaking condition (100 rpm). Biomass production in culture filtrates was analysed by determining mycelial dry weight post 3 days of inoculation.

\section{Detached leaf bioassay}

B. cinerea conidia were collected from 15 days old PDA plates with distilled water and filtered to remove the mycelial debris. Four leaves of 3-week-old $A$. thaliana ecotype Colombia-0 (Col-0) plants, grown in a Percival growth chamber (CLF plant climates, $\mathrm{GmbH}$, Germany) with growth conditions described before [32,33], were detached from each plant and placed on water agar plate with petiole inserted in agar. A $5 \mu \mathrm{l}$ droplet of conidial suspension $\left(1 \mathrm{e}+06\right.$ conidia $\left.\mathrm{ml}^{-1}\right)$ of $C$. rosea WT, deletion or complemented strains were inoculated on the adaxial surface of the leaf, dried for $30 \mathrm{~min}$ and reinoculated with equal conidial concentration of $B$. cinerea at the same place. Plants were kept in Percival growth chambers and high humidity was maintained by sealing the plates with parafilm. The diameter of necrotic lesions was measured post $56 \mathrm{~h}$ of inoculation under the microscope using a DeltaPix camera and software (DeltaPix, Denmark). Bioassay experiments were performed in 3 biological replicates and each replicate consisted of 16 leaves from 4 plants for each treatment. The experiment was repeated 2 times.

\section{Arabidopsis thaliana root colonization assay}

Surface sterile seeds of $A$. thaliana ecotype Col-0 were grown on 0.2X MS agar plates. Plates were settled vertically, to avoid burial of roots in medium, in a Percival growth chamber (CLF plant climates, $\mathrm{GmbH}$, Germany) with a growth conditions described before [32,33]. C. rosea conidia $(5 e+04)$ were inoculated under sterile conditions to the middle of 10 days old seedling roots and were co-cultivated for 5 days. Water inoculated roots were treated as control. For each set of experiments 5 biological replicates with 10 seedlings per replicate were used. To quantify the root colonization, detached roots were washed carefully with water, surface sterilized with $2 \% \mathrm{NaOCl}$ for $1 \mathrm{~min}$, weighed, and homogenised in $2 \mathrm{ml}$ sterile water. Serial dilutions were plated on PDA plates to count colony forming units. The complementation strains $\Delta H y d 1+$ and $\Delta H y d 3+$ and four independent $H y d 1 H y d 3$ mutant strains were included in all phenotype analyses to exclude the possibility that phenotypes derive from ectopic insertions. No significant difference in data of analysed phenotypes were found between four independent Hyd1Hyd3 mutant strains, therefore data from one representative deletion strain are presented in the figures.

\section{Statistical analysis}

Analysis of variance (ANOVA) was performed on gene expression and phenotype data using a General Linear Model approach implemented in Statistica version 10 (StatSoft, Tulsa, OK). Pairwise comparisons were made using the Tukey-Kramer method at the 95\% significance level.

\section{Additional file}

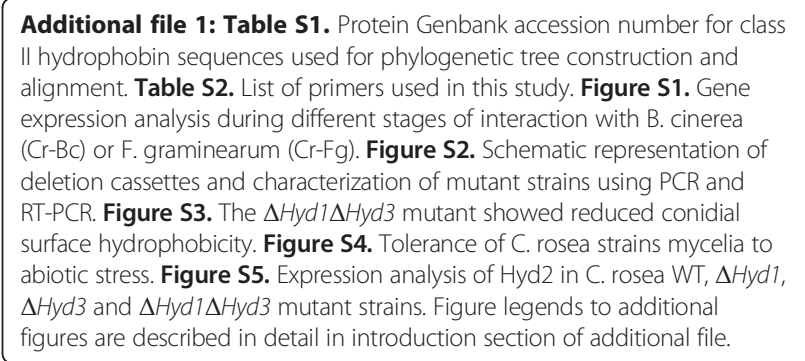

Competing interests

The authors declare that they have no competing interests.

\section{Authors' contributions}

Conceived and designed the experiments: MD DFJ MK. Performed the experiments: MD. Analyzed the data: MD MK. Contributed reagents/materials/ analysis tools: DFJ MK. Wrote the paper: MD MK. All authors read and approved the manuscript.

\section{Acknowledgements}

This work was financially supported by the Department of Forest Mycology and Plant Pathology, Swedish Research Council for Environment, Agricultural Sciences and Spatial Planning (FORMAS, grant number 229-2009-1530 and 229-2012-1288), and Danish Agency for Science, Technology and Innovation (DSF grant number 09-063108/DSF).

Received: 18 November 2013 Accepted: 27 January 2014

Published: 31 January 2014

\section{References}

1. Wessels JG: Hydrophobins: proteins that change the nature of the fungal surface. Adv Microb Physiol 1997, 38:1-45.

2. Wosten HA: Hydrophobins: multipurpose proteins. Ann Rev Microbiol 2001, 55:625-646.

3. Linder MB, Szilvay GR, Nakari-Setala T, Penttila ME: Hydrophobins: the protein-amphiphiles of filamentous fungi. FEMS Microbiol Rev 2005, 29:877-896. 
4. Jensen BG, Andersen MR, Pedersen MH, Frisvad JC, Sondergaard I: Hydrophobins from Aspergillus species cannot be clearly divided into two classes. BMC Res Notes 2010, 3:344.

5. Seidl-Seiboth V, Gruber S, Sezerman U, Schwecke T, Albayrak A, Neuhof T, von Dohren H, Baker SE, Kubicek CP: Novel hydrophobins from Trichoderma define a new hydrophobin subclass: protein properties, evolution, regulation and processing. J Mol Evol 2011, 72:339-351.

6. Whiteford JR, Spanu PD: Hydrophobins and the interactions between fungi and plants. Mol Plant Pathol 2002, 3:391-400.

7. Bayry J, Aimanianda V, Guijarro Jl, Sunde M, Latgé J-P: Hydrophobins-unique fungal proteins. PLOS Pathol 2012, 8:e1002700.

8. Talbot NJ, Kershaw MJ, Wakley GE, De Vries O, Wessels J, Hamer JE: MPG1 encodes a fungal hydrophobin involved in surface interactions during infection-related development of Magnaporthe grisea. Plant Cell 1996, 8:985-999.

9. Kim S, Ahn IP, Rho HS, Lee YH: MHP1, a Magnaporthe grisea hydrophobin gene, is required for fungal development and plant colonization. Mol Microbiol 2005, 57:1224-1237.

10. Zhang S, Xia YX, Kim B, Keyhani NO: Two hydrophobins are involved in fungal spore coat rodlet layer assembly and each play distinct roles in surface interactions, development and pathogenesis in the entomopathogenic fungus, Beauveria bassiana. Mol Microbiol 2011, 80:811-826.

11. Sevim A, Donzelli BG, Wu D, Demirbag Z, Gibson DM, Turgeon BG: Hydrophobin genes of the entomopathogenic fungus, Metarhizium brunneum, are differentially expressed and corresponding mutants are decreased in virulence. Curr Genet 2012, 58:79-92.

12. Fuchs U, Czymmek KJ, Sweigard JA: Five hydrophobin genes in Fusarium verticillioides include two required for microconidial chain formation. Fungal Genet Biol 2004, 41:852-864.

13. Mosbach A, Leroch M, Mendgen KW, Hahn M: Lack of evidence for a role of hydrophobins in conferring surface hydrophobicity to conidia and hyphae of Botrytis cinerea. BMC Microbiol 2011, 11:10.

14. Sutton JC, Li D, Peng G, Yu H, Zhang P, Valdebeneito-Sanhueza RM: Gliocladium roseum a versatile adversary of Botrytis cinerea in crops. Plant Dis 1997, 81:316-328.

15. Li GQ, Huang HC, Acharya SN, Erickson RS: Biological control of blossom blight of alfalfa caused by Botrytis cinerea under environmentally controlled and field conditions. Plant Dis 2004, 88:1246-1251.

16. Luongo L, Galli M, Coraz L, Meekes E, De Haas L, Plas LV, Köhl J: Potential of fungal antagonists for biocontrol of Fusarium spp. in wheat and maize through competition in crop debris. Biocontr Sci Tech 2005, 15:229-242.

17. Koch E, Schmitt A, Stephan D, Kromphardt C, Jahn M, Krauthausen H, Forsberg G, Werner S, Amein T, Wright SAI, Tinivella F, Gullino ML, Roberts SJ, Wolf J, Groot SPC: Evaluation of non-chemical seed treatment methods for the control of Alternaria dauci and A. radicina on carrot seeds. Eur J Plant Pathol 2010, 127:99-112.

18. Rodriguez MA, Cabrera G, Gozzo FC, Eberlin MN, Godeas A: Clonostachys rosea BAFC3874 as a Sclerotinia sclerotiorum antagonist: mechanisms involved and potential as a biocontrol agent. J App/ Microbiol 2011, 110:1177-1186.

19. Toledo AV, Virla E, Humber RA, Paradell SL, Lastra CC: First record of Clonostachys rosea (Ascomycota: Hypocreales) as an entomopathogenic fungus of Oncometopia tucumana and Sonesimia grossa (Hemiptera: Cicadellidae) in Argentina. J Invertebr Pathol 2006, 92:7-10.

20. Zhang L, Yang J, Niu Q, Zhao X, Ye F, Liang L, Zhang KQ: Investigation on the infection mechanism of the fungus Clonostachys rosea against nematodes using the green fluorescent protein. Applied Microbiol Biotechnol 2008, 78:983-990.

21. Zou CG, Tu HH, Liu XY, Tao N, Zhang KQ: PacC in the nematophagous fungus Clonostachys rosea controls virulence to nematodes. Environ Microbiol 2010, 12:1868-1877.

22. Roberti R, Eva Z, Flamigni F, De Vero L, Cesari A: Antagonistic fungi producing hydrolytic enzymes, active in degrading the cell wall of some foot rot pathogens (Fusarium spp.) of wheat. J Plant Dis Protect 2002, 109:101-108.

23. Lübeck M, Knudsen IMB, Jensen B, Thrane U, Janvier C, Jensen DF: GUS and GFP transformation of the biocontrol strain Clonostachys rosea IK726 and the use of these marker genes in ecological studies. Mycol Res 2002, 106:818-826.

24. Chatterton S, Jayaraman J, Punja ZK: Colonization of cucumber plants by the biocontrol fungus Clonostachys rosea f. catenulata. Biol control 2008 46:267-278
25. Roberti R, Veronesi A, Cesaria A, Casconeb A, Berardinob ID, Bertinib L, Caruso C: Induction of PR proteins and resistance by the biocontrol agent Clonostachys rosea in wheat plants infected with Fusarium culmorum. Plant Sci 2008, 175:339-347.

26. Askolin S, Penttila M, Wosten HA, Nakari-Setala T: The Trichoderma reese hydrophobin genes $h f b 1$ and $h f b 2$ have diverse functions in fungal development. FEMS Microbiol Lett 2005, 253:281-288.

27. Bailey MJ, Askolin S, Horhammer N, Tenkanen M, Linder M, Penttila M, Nakari-Setala T: Process technological effects of deletion and amplification of hydrophobins I and II in transformants of Trichoderma reesei. Appl Microbiol Biotechnol 2002, 58:721-727.

28. Viterbo A, Chet I: TasHyd1, a new hydrophobin gene from the biocontrol agent Trichoderma asperellum, is involved in plant root colonization. Mol Plant Pathol 2006, 7:249-258.

29. Kubicek CP, Baker S, Gamauf C, Kenerley CM, Druzhinina IS: Purifying selection and birth-and-death evolution in the class II hydrophobin gene families of the ascomycete Trichoderma/Hypocrea. BMC Evol Biol 2008, 8:4.

30. Lora JM, Pintor-Tora JA, Benítez T, Romero LC: Qid3 protein links plant bimodular proteins with fungal hydrophobins. Mol Microbiol 1995 18:377-382

31. Dubey MK, Ubhayasekera W, Sandgren M, Jensen DF, Karlsson M: Disruption of the Eng18B ENGase gene in the fungal biocontrol agent Trichoderma atroviride affects growth, conidiation and antagonistic ability. PLOS One 2012, 7:e36152.

32. Dubey MK, Broberg A, Sooriyaarachchi S, Ubhayasekera W, Jensen DF, Karlsson $\mathrm{M}$ : The glyoxylate cycle is involved in pleotropic phenotypes, antagonism and induction of plant defence responses in the fungal biocontrol agent Trichoderma atroviride. Fungal Genet Biol 2013, 58-59:33-41.

33. Dubey MK, Broberg A, Jensen DF, Karlsson M: Role of the methylcitrate cycle in growth, antagonism and induction of systemic defence responses in the fungal biocontrol agent Trichoderma atroviride. Microbiology 2013, 159:2492-2500.

34. Whiteford JR, Spanu PD: The hydrophobin HCf-1 of Cladosporium fulvum is required for efficient water-mediated dispersal of conidia. Fungal Genet Biol 2001, 32:159-168.

35. Fang W, Bidochka MJ: Expression of genes involved in germination, conidiogenesis and pathogenesis in Metarhizium anisopliae using quantitative real-time RT-PCR. Mycol Res 2006, 110:1165-1171.

36. Linder MB: Hydrophobins: protein that self assemble at interfaces. Curr Opin Colloid Interface Sci 2009, 14:356-363.

37. Mikus M, Hatvani L, Neuhof T, Komon-Zelazowska M, Dieckmann R, Schwecke T, Druzhinina IS, von Dohren H, Kubicek CP: Differential regulation and posttranslational processing of the class II hydrophobin genes from the biocontrol fungus Hypocrea atroviridis. Appl Environ Microbiol 2009, 75:3222-3229.

38. Mey G, Correia T, Oeser B, Kershaw MJ, Garre V, Arntz C, Talbot NJ, Tudzynski P: Structural and functional analysis of an oligomeric hydrophobin gene from Claviceps purpurea. Mol Plant Pathol 2003, 4:31-41.

39. Bowden CG, Smalley E, Guries RP, Hubbes M, Temple B, Horgen PA: Lack of association between cerato-ulmin production and virulence in Ophiostoma novo-ulmi. Mol Plant-Microbe Interact 1996, 9:556-564.

40. van Wetter MA, Wosten HA, Wessels JG: SC3 and SC4 hydrophobins have distinct roles in formation of aerial structures in dikaryons of Schizophyllum commune. Mol Microbiol 2000, 36:201-210.

41. Wösten HA, Schuren FJ, Wessels JG: Interfacial self-assembly of a hydrophobin into an amphipathic protein membrane mediates fungal attachment to hydrophobic surfaces. EMBO J 1994, 13:5848-5854.

42. Bork P, Letunic I, Doerks T: SMART 6: recent updates and new developments. Nucleic Acids Res 2009, 37:D229-D232.

43. Quevillon E, Silventoinen V, Pillai S, Harte N, Mulder N, Apweiler R, Lopez R: InterProScan: protein domains identifier. Nucleic Acids Res 2005 33:W116-W120.

44. Marchler-Bauer A, Lu S, Anderson JB, Chitsaz F, Derbyshire MK, DeWeese-Scott C, Fong JH, Geer LY, Geer RC, Gonzales NR, Gwadz M, Hurwitz DI, Jackson JD, Ke Z, Lanczycki CJ, Lu F, Marchler GH, Mullokandov M, Omelchenko MV, Robertson CL, Song JS, Thanki N, Yamashita RA, Zhang D, Zhang N, Zheng C, Bryant SH: CDD: a conserved domain database for the functional annotation of proteins. Nucleic Acids Res 2011, 39:D225-229.

45. Larkin MA, Blackshields G, Brown NP, Chenna R, McGettigan PA, McWilliam H, Valentin F, Wallace IM, Wilm A, Lopez R, Thompson JD, Gibson TJ, Higgins DG Clustal W and Clustal X version 2.0. Bioinformatics 2007, 23:2947-2948. 
46. Petersen TN, Brunak S, von Heijne G, Nielsen H: SignalP 4.0: Discriminating signal peptides from transmembrane regions. Nat Methods 2011, 8:785-786.

47. Gasteiger E, Gattiker A, Hoogland C, Ivanyi I, Appel RD, Bairoch A: ExPASy: the proteomics server for in-depth protein knowledge and analysis. Nucleic Acids Res 2003, 31:3784-3788.

48. Anisimova M, Gil M, Dufayard JF, Dessimoz C, Gascuel O: Survey of branch support methods demonstrates accuracy, power, and robustness of fast likelihood-based approximation schemes. Syst Biol 2011, 60:685-699.

49. Le SQ, Gascuel O: An improved general amino acid replacement matrix. Mol Biol Evol 2008, 25:1307-1320.

50. Tzelepis GD, Melin P, Jensen DF, Stenlid J, Karlsson M: Functional analysis of glycoside hydrolase family 18 and 20 genes in Neurospora crassa. Fungal Genet Biol 2012, 49:717-730.

51. Mamarabadi M, Jensen B, Jensen DF, Lubeck M: Real-time RT-PCR expression analysis of chitinase and endoglucanase genes in the three-way interaction between the biocontrol strain Clonostachys rosea IK726, Botrytis cinerea and strawberry. FEMS Microbiol Lett 2008, 285:101-110.

52. Pfaffl MW: A new mathematical model for relative quantification in real-time RT-PCR. Nucleic Acids Res 2001, 29:2002-2007.

53. Nygren CMR, Eberhardt U, Karlsson M, Parrent JL, Lindahl BD, Taylor AFS: Growth on nitrate and occurrence of nitrate reductase-encoding genes in a phylogenetically diverse range of ectomycorrhizal fungi. New Phytol 2008, 180:875-889.

54. Lorang JM, Tuori RP, Martinez JP, Sawyer TL, Redman RS, Rollins JA, Wolpert TJ, Johnson KB, Rodriguez RJ, Dickman MB, Ciuffetti LM: Green fluorescent protein is lighting up fungal biology. Appl Environ Microb 2001, 67:1987-1994.

55. Kück U, Hoff B: Application of the nourseothricin acetyltransferase gene (nat1) as dominant marker for the transformation of filamentous fungi. Fungal Genet News Lett 2006, 93:9-11.

56. Karimi M, De Meyer B, Hilson P: Modular cloning in plant cells. Trends Plant Sci 2005, 10:103-105.

57. Utermark J, Karlovsky P: Genetic transformation of filamentous fungi by Agrobacterium tumefaciens. Nature Protocols Online 2008, 83. doi:10.1038/ nprot.2008.1083.

doi:10.1186/1471-2180-14-18

Cite this article as: Dubey et al:: Hydrophobins are required for conidial hydrophobicity and plant root colonization in the fungal biocontrol agent Clonostachys rosea. BMC Microbiology 2014 14:18,

\section{Submit your next manuscript to BioMed Central and take full advantage of:}

- Convenient online submission

- Thorough peer review

- No space constraints or color figure charges

- Immediate publication on acceptance

- Inclusion in PubMed, CAS, Scopus and Google Scholar

- Research which is freely available for redistribution 